\title{
Comparison of Quantitative Hepatitis B Virus DNA Real Time PCR (RT-PCR) with Reverse Transcription PCR (rt-PCR)
}

\section{Ghazala Rubi ${ }^{*}$, Sahr Malik ${ }^{2}$ and Mubeshra Jamil ${ }^{2}$}

${ }^{1}$ Director Research, Post Graduate Medical Institute, Lahore, Pakistan

${ }^{2}$ Research Assistant Lahore General Hospital), Lahore, Pakistan

*Corresponding Author: Ghazala Rubi, Director Research, Post Graduate Medical Institute, Lahore, Pakistan.

Received: April 27, 2019; Published: July 03, 2019

DOI: $10.31080 /$ ASGIS.2019.02.0054

\begin{abstract}
Background: Hepatitis B Virus Serum DNA is a reliable and useful marker for the diagnosis and monitoring of HBV infection. The limitation of HBV DNA serum assays are that they are not economical and also lack uniformity and standardization. Therefore, there is a need for a more reliable and economical marker for the diagnosis of HBV. HBsAg quantitation is one such substitute serological marker. The objective of this study is to compare the serum HBV DNA quantitative real time PCR with HBV reverse transcription PCR (rt-PCR)

Methods: Patients diagnosed with HBV from all the departments of hospital were enrolled in this study. Patients, with undetectable Hepatitis B virus and those co-infected with HIV or HCV, were excluded from the study. All patients were tested for HBV DNA PCR and serological markers like HBeAg, HBsAg. HBsAg quantification was performed using conventional ELISA immunoassay. The comparison between HBV DNA (RT-PCR) and (rt-PCR) quantitation was done by using Chi-square. SPSS was used for statistical analysis and $\mathrm{P}$-value of $<0.05$ was considered significant.

Results: A total of 661 patients were included in this study. Out of these 661 patients, 373 serum samples were analyzed using HBV RT-PCR while 281 by HBV rt-PCR. The percentage of female patients in the group of HBV RT-PCR was $38.9 \%$ and $32.7 \%$ in the group of HBV rt-PCR. The mean age of patients in the group of HBV RT-PCR was 33.01 years and 34.61 years in the group of HBV rt-PCR. In the group of HBV RT-PCR, the mean ALT level was $57.6 \mathrm{u} / \mathrm{l}$ and 51.00 in the group of HBV rt-PCR. In the group of HBV RT-PCR 16.5\% $(n=61)$ and $8.9 \%(n=33)$ were HBeAg positive. In the group of HBV RT-PCR 94.9\% and in the group of HBV rt-PCR, 73.2\% were HBsAg positive. The mean of HBV DNA positive in the group of HBV RT-PCR was $44.3 \%$ and in the group of HBV rt-PCR was $14.6 \%$. The levels of HBV DNA (positive) were considerably higher in HBV RT-PCR patients as compared to HBV rt-PCR patients (164 versus 54; $\mathrm{p}=0.001$ ). Neither HBsAg levels nor HBeAg levels were significant ( $\mathrm{p}=0.573,0.057)$. Therefore, this study concludes that HBV RealTime RT-PCR is a better option for the diagnosis of HBV DNA PCR as clinically significant results obtained from this test. HBV RT-PCR is a useful and significant technology for diagnosis of HBV DNA PCR, it must be used appropriately.

Conclusions: There is a significant difference between HBV DNA Real-Time PCR (RT-PCR) and HBV DNA reverse transcription PCR (rt-PCR) for the diagnosis of patients with hepatitis B virus but not in HBsAg and HBeAg.
\end{abstract}

Keywords: HBV; RT PCR; Reverse Transcription PCR; HBeAg; HBsAg Quantitation

\section{Introduction}

Hepatitis B virus is a significant global public health problem [1]. HBV is a double stranded DNA virus that belongs to the Hepadnaviridae family [2]. HBV has serious health consequences regard- ing both acute and chronic infection leading to liver cirrhosis and liver failure or hepatocellular carcinoma [3]. Globally, around 400 million people are chronically infected with HBV, $15 \%$ to $40 \%$ are at the risk of having serious complications of liver such as cirrhosis 
and hepatocellular carcinoma (HCC), several will die prematurely [4]. Prevalence of HBV infection varies around the world [5]. Estimates of World Health Organization (WHO) suggests that more than 2 billion people have been infected with HBV worldwide, approximately 3 million individuals have persistent infection and risk of serious illness that may lead to death, while 1 million people die every year worldwide from (HCC) and liver failure [6]. The prevalence of HBV in endemic regions like Africa, China, and Southeast Asia (excluding Japan) is very high. Approximately 8\% population are chronic HBV carriers and around 60 to $80 \%$ population are at the lifetime risk of this infection [7]. Pakistan is one of the highly endemic country with around 9 million people have been diagnosed with HBV and the infection rate is rising alarmingly [8]. Therefore, control of HBV infection is an important goal for public health.

Serum HBV DNA is the most reliable marker to detect individuals with high viral replication, on CHB therapy patients $[9,10]$. However, there are several limitations to measure serum HBV DNA. Various commercial kits available for the diagnosis of HBV; provide conflicting quantitative results and have different linear ranges. Repeated assays done to monitor a patient on antivirals on the same platform. Henceforth, there is a definite need of a monitoring tool which is reliable, economical and easy to perform. HBsAg, quantitation is a recent serological marker being assessed. Although HBsAg is not a new assay but its fully automated version has recently been introduced. This method is based on ELISA [11].

Several studies have described clinical utility of HBsAg quantitation, but there are very few studies on the comparison of these two markers and the results which are available are conflicting [12-14].

\section{Methods}

The study was carried out from February to July 2018. All consecutive patients of Chronic Hepatitis B attending the outpatient clinic were included. Following patients were excluded:

- $\quad$ Patients with undetectable HBV DNA levels

- $\quad$ Patients with co-infection with HCV, human immunodeficiency virus (HIV) or hepatitis D virus (HDV)

- $\quad$ Patients undergoing treatment of HBV

- $\quad$ Patients who did not give consent.
Clinical evaluation

Clinical histories of all the patients were taken thoroughly. The sera of all the study subjects were tested for routine HBV markers HBeAg, HBsAg. All sera were subjected to RT-PCR (Real-time polymerase reaction) to detect HBV DNA. ELISA Immunoassay by Alfa diagnostics was used for the HBsAg quantification.

\section{HBV DNA quantitation}

HBV DNA quantitation was performed on patient's plasma by using CFX Connect real-time PCR detection system (BIORAD) according to manufacturer's protocol. Results were expressed in IU/ $\mathrm{ml}$.

\section{HBsAg quantitation}

HBsAg levels of subjects sera were measured by using, ELISA immunoassay, ETI Max 3000 (Diasorin) assay according to the manufacturer's protocol and the results were expressed in IU/ml. This assay is calibrated as per WHO standards and allows the quantitation of HBsAg from 0.05 to $250 \mathrm{IU} / \mathrm{ml}$. A concentration greater than $0.05 \mathrm{IU} / \mathrm{ml}$ was considered HBsAg positive. The samples with an HBsAg level higher than $250 \mathrm{IU} / \mathrm{ml}$ required a 1:500 dilution with the diluent as per the manufacturer protocol and the exact concentration of HBsAg was measured.

\section{Reverse Transcription PCR (rt-PCR)}

Statistical analysis

Quantitative variables were expressed as median with range and qualitative variables were expressed as numbers with percentage. Chi-square was used to determine relationship between HBV DNA (RT-PCR) and HBV DNA (rt-PCR). Statistical analysis was done using SPSS and $\mathrm{p}$ value of $<0.05$ was considered significant.

\section{Results}

A total of 661 patients were included in this study. Out of these 661 patients, 373 serum samples were analyzed by HBV RT-PCR while 281 by HBV rt-PCR. The percentage of female patients in group of HBV RT-PCR was $38.9 \%$ and $32.7 \%$ in group of HBV rtPCR. The mean age of patients in group of HBV RT-PCR was 33.01 years and 34.61 years in group of HBV rt-PCR. In group of HBV RTPCR, the mean ALT level was $57.6 \mathrm{u} / \mathrm{l}$ and 51.00 in group of HBV rt-PCR. In group of HBV RT-PCR 16.5\% (n=61) and 8.9\% (n=33) were HBeAg positive. In group of HBV RT-PCR $94.9 \%$ and in group of HBV rt-PCR $73.2 \%$ were HBsAg were positive. The mean of HBV 
DNA positive in group of HBV RT-PCR was $44.3 \%$ and in group of HBV rt-PCR was $14.6 \%$. The levels of HBV DNA (positive) were considerably higher in HBV RT-PCR patients as compared to HBV rt-PCR patients (164 versus $54 ; \mathrm{p}=0.001$ ). Neither HBsAg levels nor HBeAg levels were significant ( $p=0.573,0.057)$. Therefore, this study concludes that HBV Real Time RT-PCR is a better option for the diagnosis of HBV DNA PCR as clinically significant results obtained from this test. HBV RT-PCR is a useful and significant technology for diagnosis of HBV DNA PCR, it must be used appropriately.

\begin{tabular}{|l|c|c|}
\hline Baseline variables & (RT-PCR) & (rt-PCR) \\
\hline Mean Age & $33.01 \pm 0.770$ & $34.61 \pm 0.847$ \\
\hline Males $\%$ & $60.8 \%$ & $45.1 \%$ \\
\hline HBeAg Positive $\%$ & $16.5 \%$ & $8.9 \%$ \\
\hline ALT U/L & $57.6 \pm 8.643$ & $51.00 \pm 5.607$ \\
\hline HBV DNA POSITIVE $\%$ & $44.3 \%$ & $14.6 \%$ \\
\hline
\end{tabular}

Table 1: Baseline variables.

The mean ALT level was 57.6 U/L in group of HBV RT-PCR while, 51.00 in group of HBV rt-PCR. $16.5 \%(n=61)$ in group of HBV RTPCR while, $8.9 \%(n=33)$ in group of HBV rt-PCR were HBeAg positive. $94.9 \%(n=351)$ in group of HBV RT-PCR while, 73.2\% $(n=271)$ in group of HBV rt-PCR were HBsAg positive. Mean HBV DNA Positive $44.3 \%$ in group of HBV RT-PCR while, $14.6 \%$ in group of HBV rt-PCR.

\begin{tabular}{|c|c|c|c|}
\hline Sub group & HBV RT-PCR & HBV rt-PCR & P-value \\
\hline \multicolumn{4}{|l|}{ HBsAg } \\
\hline Positive & 351 & 271 & \multirow[t]{2}{*}{0.573} \\
\hline Negative & 15 & 15 & \\
\hline \multicolumn{4}{|l|}{$\mathrm{HBeAg}$} \\
\hline \multirow{2}{*}{$\begin{array}{l}\text { Positive } \\
\text { Negative }\end{array}$} & 61 & 33 & \multirow[b]{2}{*}{0.057} \\
\hline & 300 & 254 & \\
\hline \multicolumn{4}{|c|}{ HBV DNA } \\
\hline \multirow{2}{*}{$\begin{array}{l}\text { Positive } \\
\text { Negative }\end{array}$} & 164 & 54 & \multirow[t]{2}{*}{0.001} \\
\hline & 205 & 234 & \\
\hline
\end{tabular}

Table 2: Subgroup analysis.

HBV DNA (positive) levels were significantly higher in HBV RTPCR patients compared with HBV rt-PCR patients (164 versus 54; $\mathrm{p}=0.001$ ). Neither HBsAg levels nor HBeAg levels were significant
( $p=0.573,0.057)$. HBV Real Time RT-PCR is best for diagnosis of HBV DNA PCR. Clinical significant result obtained from such test. HBV RT-PCR has become a useful and important technology for diagnosis of HBV DNA PCR, it must be used appropriately.

\section{Discussion}

Hepatitis B virus (HBV) infection is a serious global health problem. In Asia, majority of HBV infection is acquired perinatal or in early childhood [15]. The prevalence of HBV in Pakistan is very high an estimated 09 million people are infected by Hepatitis B virus [16].

Quantitative serology done by HBsAg. HBsAg is encoded by the envelope gene, which contains three open-reading frames: the preS1, pre-S2 and S domains. There is subsequent conversion to small, medium and large forms of HBsAg proteins. Newly synthesized HBsAg proteins are secreted from the hepatocyte. Similar to the HBeAg pathway, HBsAg synthesis is separate from the viral replication pathway. Existent quantitative HBsAg serology can detect all three forms of HBsAg in the circulation. Since the introduction of quantitative HBsAg, a lot of studies have done regarding its clinical significance [17]. Many cross-sectional studies have shown significant correlation between HBsAg levels and serum HBV DNA levels $[13,18,19]$. Since, there is no study have been done to compare HBV DNA Real Time PCR (RT-PCR) with HBV DNA reverse transcription PCR (rt-PCR).

\section{Conclusion}

This study shows that there is a significant difference between HBV DNA Real Time PCR (RT-PCR) and HBV DNA reverse transcription PCR (rt-PCR) for the diagnosis of patients with hepatitis B virus but not in HBsAg and HBeAg. The limitation of the study is the small number of patients included in the study. Larger studies are required to confirm that $\mathrm{HBV}$ DNA (RT-PCR) is comparable to HBV DNA (rt-PCR).

\section{Bibliography}

1. Baig, S. "Gender disparity in infections of Hepatitis B virus". Journal of College of Physicians and Surgeons Pakistan 19.9 (2009): 598-600.

2. Col GC., et al. "Real time-PCR HBV-DNA analysis: Significance and first experience in armed forces". Medical Journal Armed Forces India 61.3 (2005): 234-237. 
3. Yang G., et al. "Association between hepatitis B virus infection and HLA-DRB1 genotyping in Shaanxi Han patients in northwestern China". Tissue Antigens 69.2 (2007): 170-175.

4. Kowdley KV., et al. "Prevalence of chronic hepatitis B among foreign-born persons living in the United States by country of origin". Hepatology 56.2 (2012): 422-433.

5. Gust I. "Epidemiology of hepatitis B infection in the Western Pacific and South East Asia". Gut 38 (1996): S18-S23.

6. Kolassery S. "Correlation of HBsAg quantitation by ELISA with serum hepatitis B virus DNA quantitative PCR in chronic hepatitis B patients". International Journal of Research in Medical Sciences 5.6 (2017): 2422-2425.

7. Lavanchy D and M Kane. "Global epidemiology of hepatitis B virus infection". Hepatitis B Virus in Human Diseases, Springer: (2016): 187-203.

8. Noorali S., et al. "Prevalence of Hepatitis B virus genotype D in females in Karachi, Pakistan". The Journal of Infection in Developing Countries 2 (2008): 373-378.

9. Martinot-Peignoux M., et al. "HBsAg quantification to optimize treatment monitoring in chronic hepatitis B patients". Liver International 35 (2015): 82-90.

10. Foster GR., et al. "Impact of direct acting antiviral therapy in patients with chronic hepatitis $\mathrm{C}$ and decompensated cirrhosis". Journal of hepatology 64.6 (2016): 1224-1231.

11. Deguchi M., et al. "Quantitation of hepatitis B surface antigen by an automated chemiluminescent microparticle immunoassay". Journal of virological methods 115.2 (2004): 217-222.

12. Kuhns MC., et al. "Lack of correlation between HBsAg and HBV DNA levels in blood donors who test positive for HBsAg and anti-HBc: implications for future HBV screening policy". Transfusion 44.9 (2004): 1332-1339.

13. Ozaras R., et al. "Correlation of quantitative assay of HBsAg and HBV DNA levels during chronic HBV treatment". Digestive diseases and sciences 53.11 (2008): 2995-2998.

14. Wursthorn K., et al. "Kinetics of hepatitis B surface antigen decline during 3 years of telbivudine treatment in hepatitis B e antigen-positive patients". Hepatology 52.5 (2010): 16111620.
15. Thakur V., et al. "Profile, spectrum and significance of HBV genotypes in chronic liver disease patients in the Indian subcontinent". Journal of gastroenterology and hepatology 17.2 (2002): 165-170.

16. Hakim S., et al. "Seroprevalence of hepatitis B and C genotypes among young apparently healthy females of Karachi-Pakistan". Libyan Journal of Medicine 3.2 (2008): 66-70.

17. Ozdil B., et al. "Negative correlation between viral load and HBsAg levels in chronic HBV-infected patients". Archives of virology 154.9 (2009): 1451-1455.

18. Kohmoto M., et al. "Quantitative detection of hepatitis B surface antigen by chemiluminescent microparticle immunoassay during lamivudine treatment of chronic hepatitis B virus carriers". Journal of medical virology 75.2 (2005): 235-239.

19. Sonneveld M., et al. "Hepatitis B surface antigen monitoring and management of chronic hepatitis B". Journal of viral hepatitis 18.7 (2011): 449-457.

\section{Volume 2 Issue 6 August 2019}

(C) All rights are reserved by Ghazala Rubi., et al. 